\title{
Roughness parameters for classification of as-built AM surfaces
}

\author{
B. Richter ${ }^{1}$, N. Blanke ${ }^{2}$, C. Werner ${ }^{2}$, F. Vollertsen ${ }^{2,3}$, F.E. Pfefferkorn ${ }^{1 *}$ \\ ${ }^{1}$ Department of Mechanical Engineering, University of Wisconsin - Madison, Madison, WI \\ 53706, USA \\ ${ }^{2}$ Bremer Institut für angewandte Strahltechnik GmbH, Bremen, 28359, Germany \\ ${ }^{3}$ University of Bremen, Bremen, 28359, Germany
}

\begin{abstract}
One of the challenges facing the industrial adoption of additively manufactured parts is the surface roughness on the as-built part. The surface roughness of parts is frequently characterized by metrics specified by international standards organizations. However, these standards list many surface metrics that can make it unclear which to use to best describe the surface. In this work, the ability of the various surface metrics to successfully classify the as-built and post-processed surfaces is studied using linear classification models. Laser polishing via remelting and manual grinding are the post-processing techniques used to smooth the as-built surface. The ability of the linear classifier to successfully categorize the various surfaces is demonstrated, and the various surface metrics are ranked according to the strength of their individual ability to classify the surfaces. This work promotes the method as a potential way to autonomously classify as-built and laser polished surfaces.
\end{abstract}

Keywords: Laser; Polishing; Additive manufacturing; Surface analysis; Identification; Topography

\section{Introduction}

Additive manufacturing is an emerging technology that enables the production of complex parts. One additive manufacturing process, powder-bed fusion, fuses powder in a layer-by-layer manner with a laser or electron beam, to form a completed part. However, the poor surface quality of the resultant parts present a challenge that must be addressed to make the parts suitable for industrial usage [1]. A variety of post-processing techniques, such as machining, vibratory abrasive polishing, electrochemical polishing, and hand finishing have been used to address and improve the as-built part's surface [2]. One method that has received increased attention in the past few years for the finishing of additive manufactured parts is laser polishing. In this technique, a laser irradiates the surface of the part and surface tension causes asperities within the molten region to smooth out leaving a better final surface finish. The technique has been demonstrated to improve the surface of additive manufactured parts on a variety of different metallic alloys, including cobalt, aluminum, steel, and titanium alloys [3]-[8]. Another approach to improving the surface finish of additive manufactured parts is through the optimization of the build process parameters [9]-[12]. 
The majority of works that discuss the surface finish of additive manufactured parts use average surface roughness, Ra, as the primary metric [13]. Ra, which is defined by the ISO 4287 standard as the arithmetic mean deviation of the assessed profile [14], is measured from a linear profile of a surface. Sa, defined by the ISO 25178 standard as the arithmetical mean height of the scale-limited surface [15], is analogous to Ra but from an areal measurement instead of a linear measurement. Similar to $\mathrm{Ra}, \mathrm{Sa}$ is the most commonly used areal surface metric; however, work has suggested reporting other metrics, like Sz (Maximum height of the of the scale-limited surface), skewness, and kurtosis when discussing additive manufactured parts [13]. Additional parameters that were highly sensitive to changes in additive manufactured part's surfaces include Vmp (Peak material volume of the scale-limited surface), Sdr (Developed interfacial area ratio of the scale-limited surface), and Spk (Reduced peak height) [13].

As additive manufactured parts are complex and often require some type of post-processing technique to improve the final surface quality, it seems reasonable to assume that some type of automation method will be used to verify the resultant surface quality. One technique for identifying groups of items is Classification, a statistical and machine learning technique. Classification has been used in a variety of industrial applications, such as classifying weld defects [16]-[18], detecting surface defects on milled parts [19], quantifying the quality of cast part surface's [20], and classifying the occurrence of defects and quality online for a powder-bed fusion process [21], [22]. However, to the author's knowledge it has not been used to distinguish between post-processed and as-built additive manufactured parts. Additionally, there are numerous surface metrics listed in the ISO standards [14], [15], but Ra and Sa are the most, and often only, reported metrics. It is likely other surface metrics are also suitable for describing additive manufactured parts. Because of that, it is of interest to define the surface metrics that are best suited for defining the unique surfaces formed from additive manufacturing and those that are best suited for distinguishing between the as-built and post-processed surfaces. However, defining 'best' at describing the surface is difficult and likely unique to a user's specific application. This work attempts to rectify that difficulty by instead studying the ability of the various surface metrics to differentiate between different types of as-built and post-processed additive manufactured surfaces using classification techniques. The results indicate that the metrics commonly used (such as Sa), are not consistently the ideal metrics for distinguishing between surfaces, and the work lists other metrics that are more suitable for the role for these surfaces.

\section{Materials and Methods}

\section{$\underline{\text { Sample Material and Building Parameters }}$}

The experiments presented in this study used a cobalt-chromium alloy (Celsit 21-P, Stellite 21 equivalent alloy) that was built using a powder-bed fusion (PBF) process (SLM 250, SLM Solutions). The alloy's composition is given in Table 1.

Table 1. Build material composition (manufacturer provided).

\begin{tabular}{ccccc}
\hline Ni (\%) & Mo (\%) & Cr (\%) & $\begin{array}{c}\text { Others } \\
(<\mathbf{3 . 3 4 8 \% )}\end{array}$ & Co (\%) \\
\hline 2.74 & 5.28 & 26.6 & B, C, Fe, P, Si & Bal. \\
\hline
\end{tabular}


The nominal powder diameter was distributed between $20 \mu \mathrm{m}$ and $53 \mu \mathrm{m}$, and the build process used a layer thickness of $100 \mu \mathrm{m}$, laser power of $110 \mathrm{~W}$, laser beam diameter of $60 \mu \mathrm{m}$, and a laser scan speed of $158 \mathrm{~mm} / \mathrm{s}$. The samples being studied were rectangular cuboids that were built upright at a 90 angle and angled at a 45 angle. The top facing surface of the 45 sample is the surface of interest for this work. The nominal size of the samples was $10 \mathrm{~mm}$ in the vertical direction (build direction) and $20 \mathrm{~mm}$ and $3.5 \mathrm{~mm}$ in the horizontal direction. An example of the geometries of the two different surfaces can be seen in Figure 1.

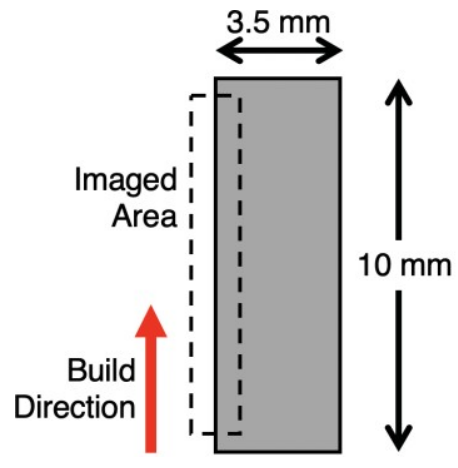

(a)

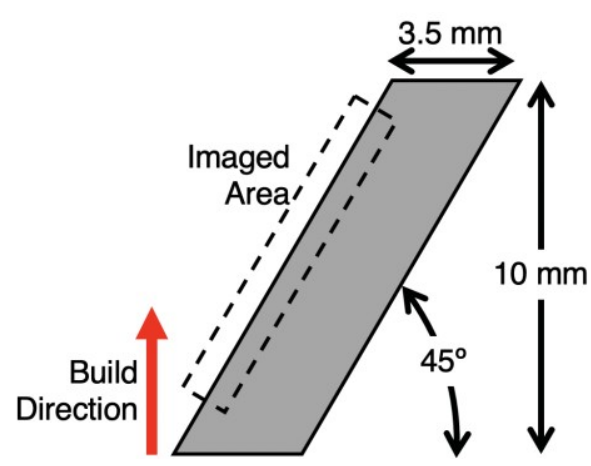

(b)

Figure 1. Example of the geometry for the (a) 90 and (b) 45 surfaces.

The surfaces of interest on the samples in this study is the side orthogonal to the build layer orientation for the 90 sample, and the surface at a build layer for the 45 sample, which is shown in Figure 1. An example of a representative surface of the as-built 90 sample and as-built 45 can be observed in Figure 2.
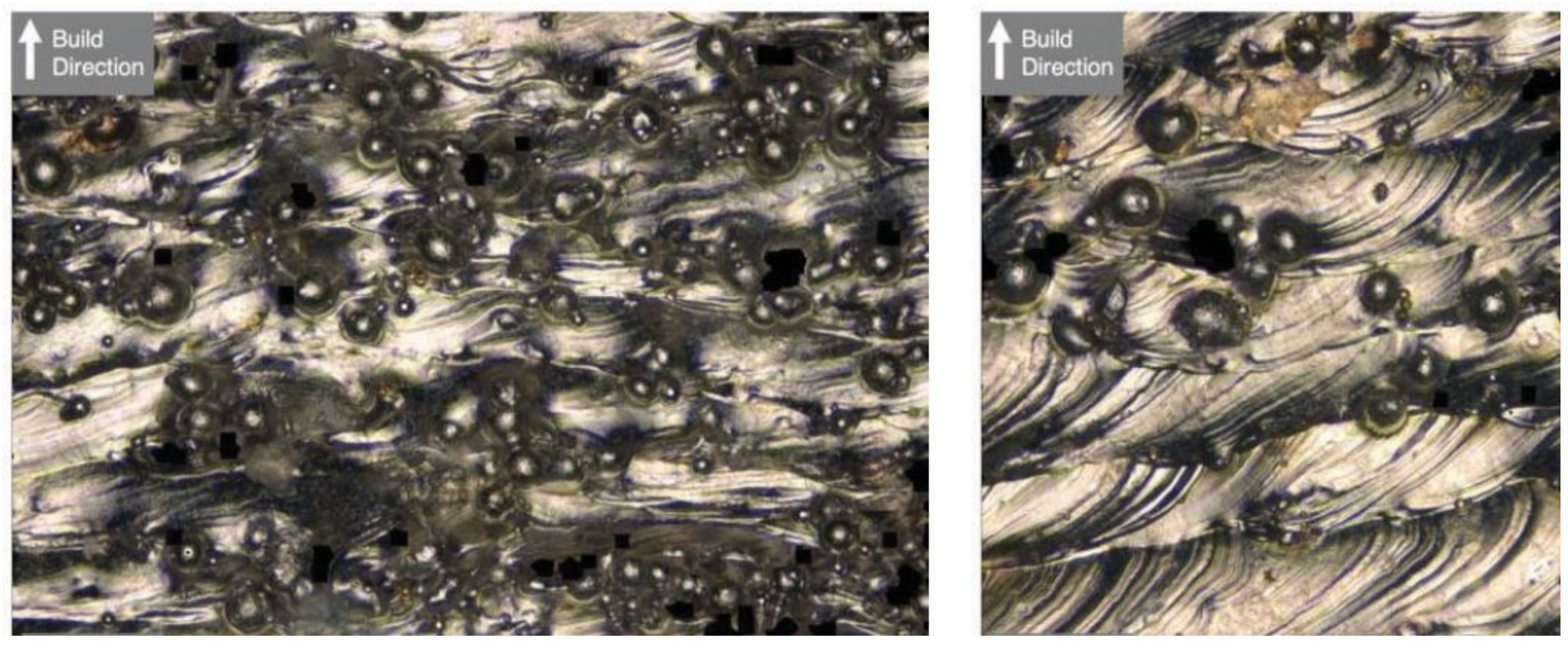

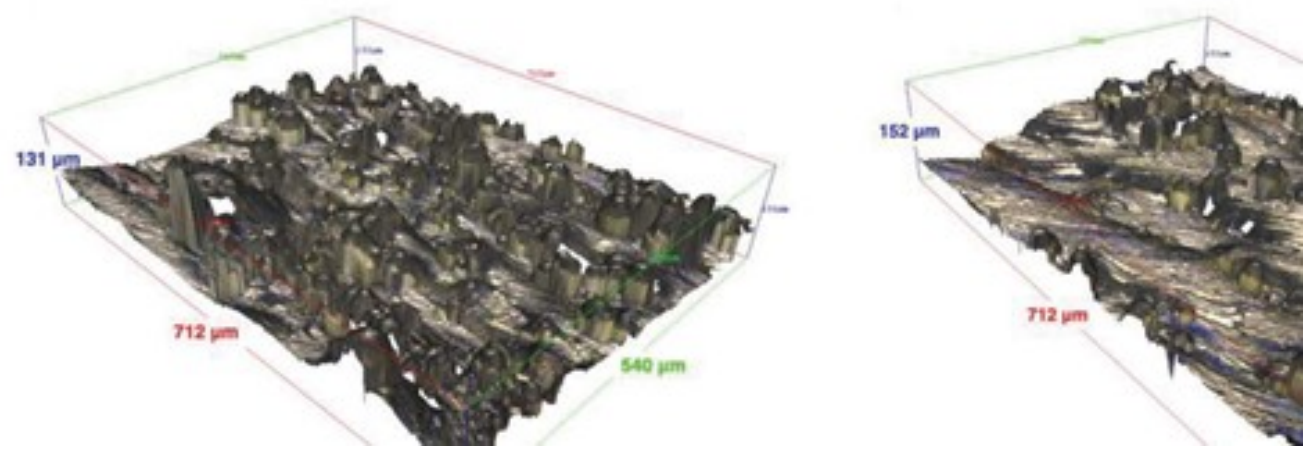

Figure 2. Top down view of representative examples of the as-built surfaces built at a (a) 90 and

(b) 45 angle and projection view of the (c) 90 and (d) 45 angle surfaces.

As Figure 2 shows, the two surfaces are relatively similar to one another with a few distinct differences. The 90 sample has a greater number of adhered particles stuck to its surface, and they are often in tighter clusters with one another than the 45 sample. The 45 sample has more pronounced large wavelength features that can be observed due to the individual build layers and the stair-step effect that occurs when building at an angle [10]. Due to the high roughness these surfaces have, they often require some type of post-processing to get to a suitable surface finish.

\section{Surface Post-Processing}

The additively manufactured surfaces were post-processed using both manual grinding and lasermicropolishing.microThemicro grindingmicro wasmicroperformedmicroonmicrothemicrosidemicroofmicroamicro90 micro sample using a grinding/polishing system and a 240-grit abrasive pad. The grinding was performed until the surface quality stopped improving for that grit size grinding paper, which took approximately two minutes.microThe laser polishing was performed on the sides of the 90 sample (surface orthogonal to the build layer) using a $200 \mathrm{~W}, 1070 \mathrm{~nm}$ fiber laser (SPI Lasers, SP-200C-W-S6-AB) that was operated in continuous-wave mode. The laser polishing conditions were based on those used in previous studies [7], and a by $1 \mathrm{~mm}$ area was polished. The nominal laser processing parameters include a beam diameter of $100 \mathrm{~m}$, a beam scanning velocity of 200 $\mathrm{mm} / \mathrm{s}$, a laser

power of $80 \mathrm{~W}$, and a spacing distance between subsequent laser lines of $60 \mathrm{~m}$. The laser traveled in the same direction for every line and scan. A total of 36 different areas were laser polished using the same polishing conditions. An example of the ground and laser polished surfaces can be seen in Figure 3. 


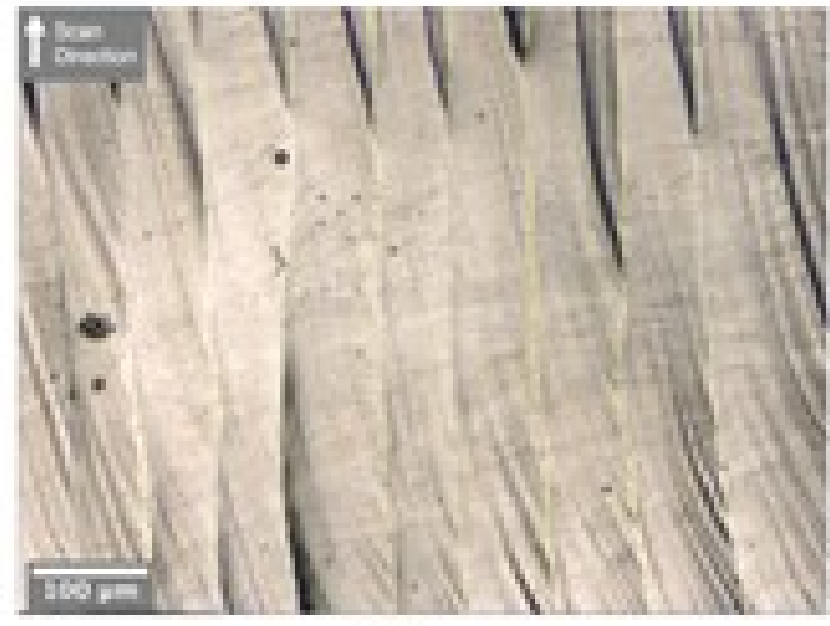

(a)

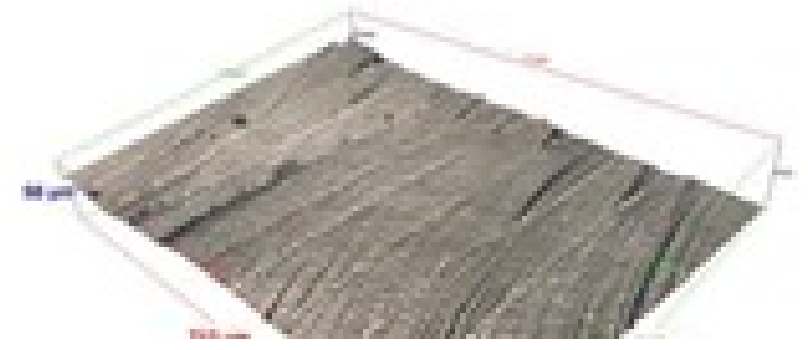

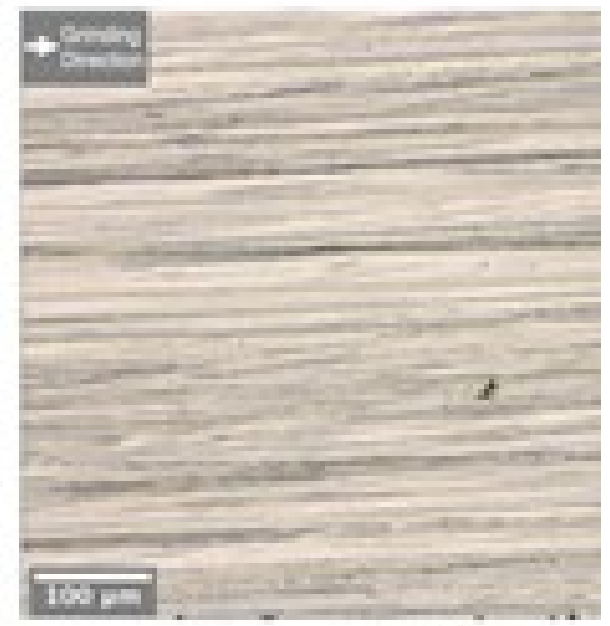

(1))

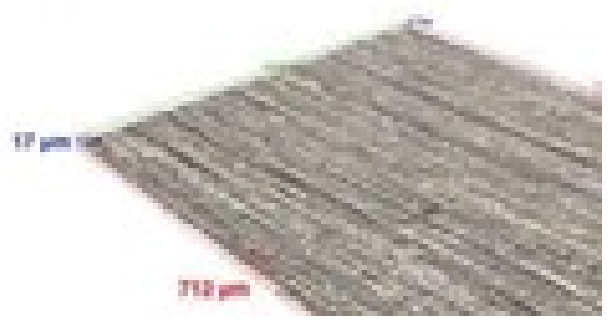

Figure 3. Top down view of the (a) laser polished and (b) ground surface and projection view of the (c) laser polished and (d) ground samples.

These surfaces are both smoother than the as-built surfaces. However, larger wavelength features can be observed in the laser polished surface (Figure 3(c)), which is due to incomplete redistribution of mass from the adhered particles during the remelting process. The ability of the laser to redistribute mass and reduce large wavelength features is strongly dependent on the processing parameters used [8]. There are also clear directional features within both of the surfaces, which correspond to either the laser scanning direction for the laser polished surfaces, or the grinding direction for the ground samples.

\section{$\underline{\text { Surface Measurement and Metrics }}$}

approximately $2 \mathrm{~mm}$ apart. From these images 20 different surface metrics were calculated and 
used for this study. The various surface metrics used in this study are listed in Table 2. More detailed information on the specific metrics can be found in the ISO 25178 standard [15].

The metrics were calculated on the primary datasets (i.e., the surface without any waviness removed) using the microscope's built-in analysis software (Alicona IF-MeasureSuite). The waviness of the surface was not filtered out due to the wide potential selection of cutoff wavelengths that could be chosen that may influence the classification; however, the overall form of the surface was removed by removing the least-square plane with a robust fitting algorithm, rendering this a S-F surface [15]. The values of the areal surface metrics for the various surfaces reported on in this work can be found in the Appendix in Table A1.

\section{Data Analysis and Classification}

For this work, two different classifications were performed. The first analysis used the surface metrics listed in Table 2 to classify the as-builtlaser polished, and ground surfaces, and the second used those same metrics to classify themicroas-built 90 and as-built 45 surfaces. Within each analysis, two different classification techniques were used: a linear discriminant analysis (LDA) classifier, and a Gaussian naive Bayes (NB) classifier. The LDA classifier attempts to categorize the various surfaces by determining the linear function that most effectively splits the categories [23]. If only one input parameter (for example, Sa) is used to classify two different surfaces, LDA determines the single value that best separates the two groups. In contrast, a Gaussian NB classifier uses a Gaussian probability distribution of the different groups to separate them [24]. For both techniques, 10-fold cross validation was used to improve confidence and reduce the chance of over-fitting. The data was analyzed, and the classification algorithms were implemented, using commercially available machine learning software (MATLAB R2018a, Statistics and Machine Learning Toolbox). The results presented in this work are reported using the error of the classification techniques for each of the individual metrics and for combinations of two metrics. The equation used to calculate the error rate is given below in Equation 1:

$$
\text { Error Rate }=\frac{\text { Incorrect Classifications }}{\text { Total Surfaces }}
$$

The error rate refers to the amount of surfaces the classifier incorrectly identified. An example of 'good' classification performance for the various surface metrics would be to have a low error rate as described by Equation 1. While various metrics from the confusion matrix can be used to assess the goodness of the classification (i.e., sensitivity, specificity, etc.), the error rate is used as there are an equal number of each group and reporting the other metrics becomes more involved for 3-way classification.

\section{$\underline{\text { Results and Discussion }}$}

\section{Motivation of Work}

This work is largely motivated by the frequent use of $\mathrm{Ra}, \mathrm{Sa}$, and $\mathrm{Sz}$ when discussing the surfaces of additively manufactured parts [13]. An example of the Sa and Sz relationship for the surfaces in this work is shown in Figure 4. The data shown are grouped according to the two different classification analyses. 


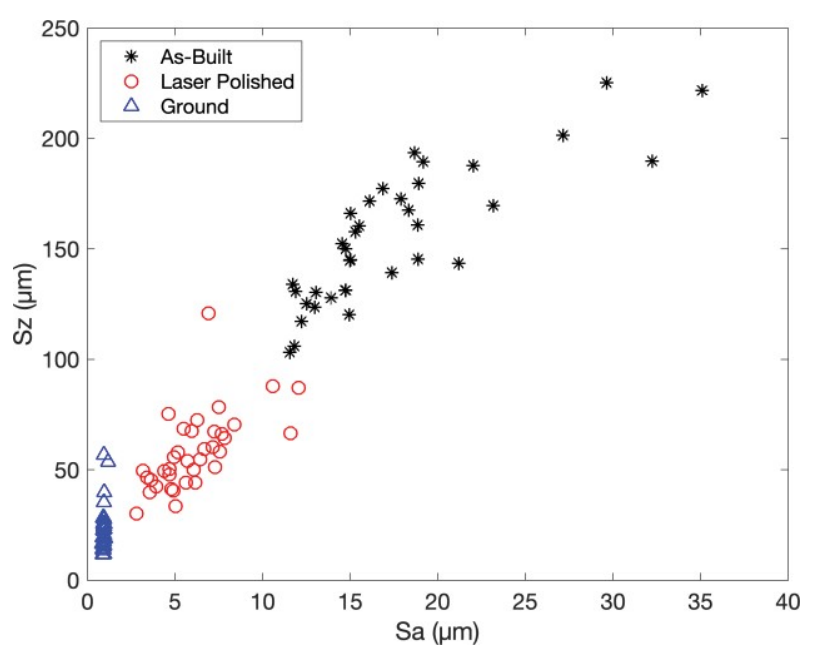

(a)

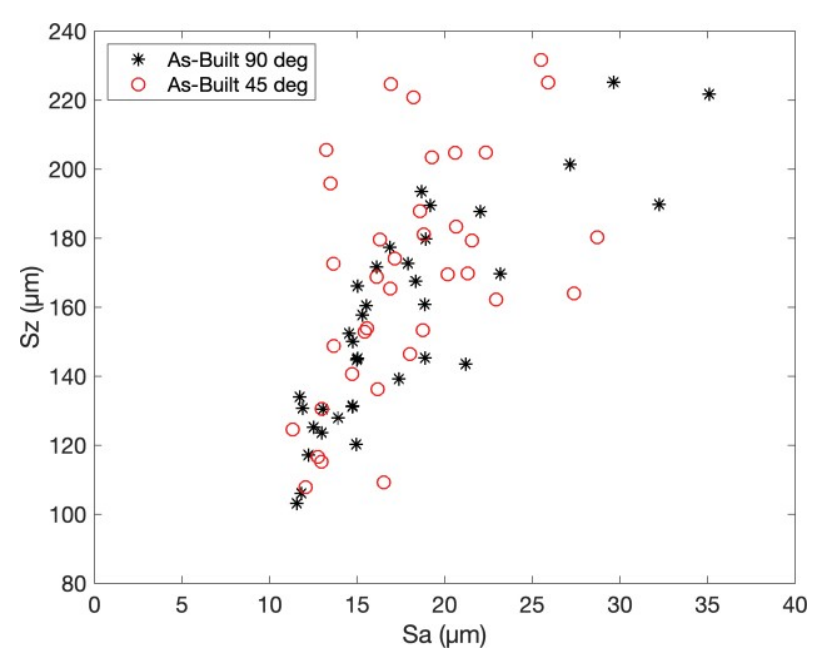

(b)

Figure 4. Sz vs. Sa plots for (a) as-built vs. post-processed and (b) as-built: 90 vs. 45 build direction.

However, as Figure 2 demonstrates, these surfaces are quite different in appearance from one another. Additionally, $\mathrm{Sa}$ and $\mathrm{Sz}$ are linearly correlated with one another, which effectively reduces the total information that is reported when both of those metrics are given. It is valuable to know which surface metrics more effectively distinguish between the different types of surfaces seen in powder-based metal additive manufacturing, which is what this study reports on.

\section{$\underline{\text { As-Built vs. Post-Processed Surfaces Classification }}$}

The error rates of the individual metrics for classifying the as-built vs. post-processed surfaces are shown in Figure 5.
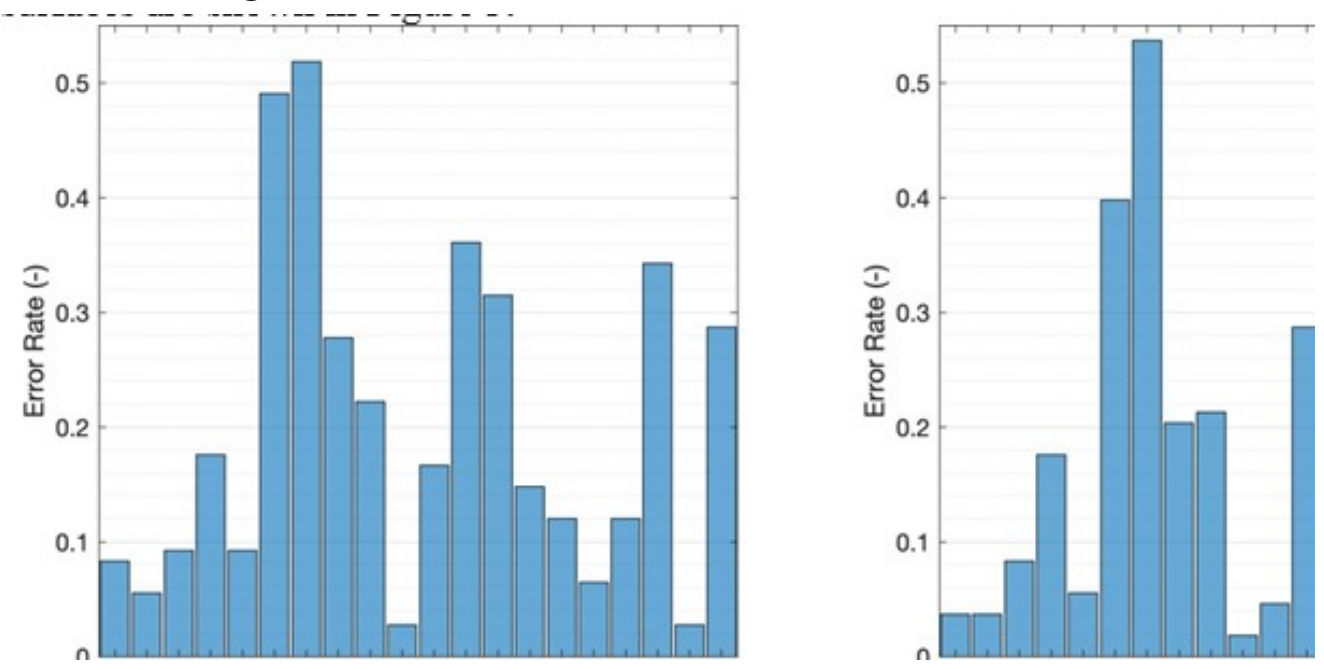

Figure 5. Error rates for the various metrics at classifying the as-built vs. post-processed surfaces using the (a) LDA classifier and (b) NB classifier.

Figure 5 shows that the most effective metrics at distinguishing the surfaces from one another for both classifications include Sk (Core height) and Sal (Autocorrelation length) with LDA error rates of 0.028 and 0.028 , respectively. Sk is derived from the bearing area curve and represents how tall the core (portion of the surface excluding peaks and valleys) is, while Sal 
represents the distance on the auto-correlation function that decays to the specified value $(0.2$ in this case) most rapidly. For this analysis, $\mathrm{Sa}$ and $\mathrm{Sz}$ are also reasonably good at distinguishing the surfaces, with NB errors rates of 0.037 and 0.056. Figure 4(a) shows this as well - Sa values can be visually seen that would reasonably separate the groups (such as $2 \mathrm{~m}$ and $10 \mathrm{~m}$ ). While this analysis tested the ability of individual metrics to distinguish the surfaces, Figure 6 shows how effective combinations of metrics are at classifying the surfaces.

Upper triangular portion are LDA results Lower triangular portion are NB I \begin{tabular}{l|l|l|l|l|l|l|l|l|l|l|l|l|l|l|l|l|l|l|}
$\mathrm{Sa}$ & 0.08 & 0.04 & 0.11 & 0.07 & 0.07 & 0.06 & 0.03 & 0.04 & 0.04 & 0.09 & 0.06 & 0.06 & 0.11 & 0.06 & 0.08 & 0.08 & 0.06 & 0.01 \\
\hline
\end{tabular} \begin{tabular}{l|l|l|l|l|l|l|l|l|l|l|l|l|l|l|l|l|l|l|l|l|l|l|l|l|} 
Sq & 0.04 & & 0.04 & 0.11 & 0.07 & 0.07 & 0.07 & 0.04 & 0.04 & 0.03 & 0.09 & 0.06 & 0.07 & 0.11 & 0.06 & 0.08 & 0.07 & 0.07 & 0.01 \\
\hline & 0.04 & 0.05 & & 0.8 & 0.08 & 0.12 & 0.06 & 0.08 & 0.09 & 0.03 & 0.10 & 0.08 & 0.12 & 0.11 & 0.12 & 0.04 & 0.07 & 0.09 & 0.01 \\
\hline
\end{tabular} \begin{tabular}{l|l|l|l|l|l|l|l|l|l|l|l|l|l|l|l|l|l|l|l|l|l|} 
Sp & 0.04 & 0.05 & & 0.08 & 0.08 & 0.12 & 0.06 & 0.08 & 0.09 & 0.03 & 0.10 & 0.08 & 0.12 & 0.11 & 0.12 & 0.04 & 0.07 & 0.09 & 0.01 \\
\hline
\end{tabular}

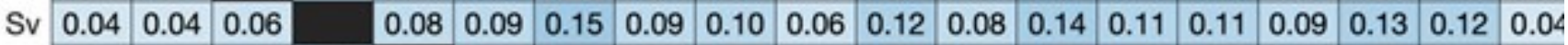

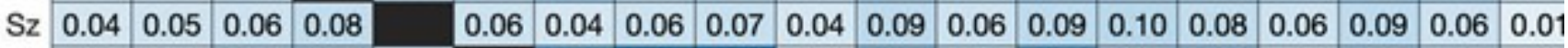

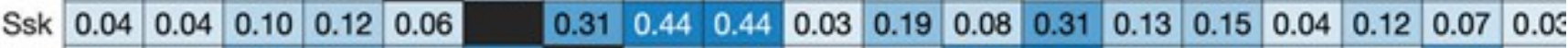

\begin{tabular}{l|l|l|l|l|l|l|l|l|l|l|l|l|l|l|l|l|l|l|l} 
Sku & 0.03 & 0.05 & 0.24 & 0.22 & 0.12 & 0.36 & & 0.26 & 0.29 & 0.01 & 0.12 & 0.37 & 0.48 & 0.14 & 0.17 & 0.05 & 0.05 & 0.33 & $0.0 \varepsilon$ \\
\hline
\end{tabular}

\begin{tabular}{|l|l|l|l|l|l|l|l|l|l|l|l|l|l|l|l|l|l|l|l|} 
Sdq & 0.01 & 0.01 & 0.06 & 0.05 & 0.04 & 0.23 & 0.26 & & 0.32 & 0.01 & 0.12 & 0.06 & 0.22 & 0.12 & 0.10 & 0.03 & 0.08 & 0.06 & 0.01 \\
\hline
\end{tabular}

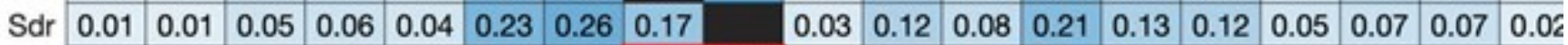

\begin{tabular}{l|l|l|l|l|l|l|l|l|l|l|l|l|l|l|l|l|l|l|l|} 
Sk & 0.02 & 0.02 & 0.02 & 0.03 & 0.03 & 0.01 & 0.03 & 0.00 & 0.00 & & 0.03 & 0.06 & 0.05 & 0.08 & 0.04 & 0.02 & 0.03 & 0.06 & 0.02 \\
\hline
\end{tabular}

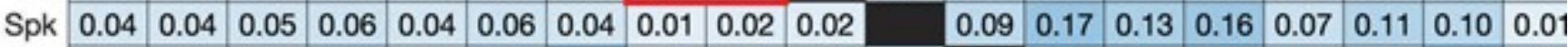

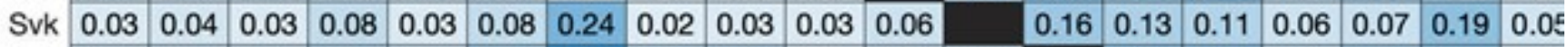

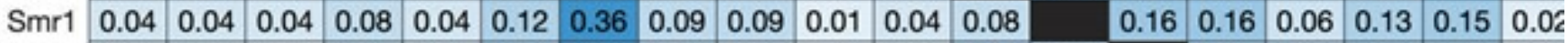

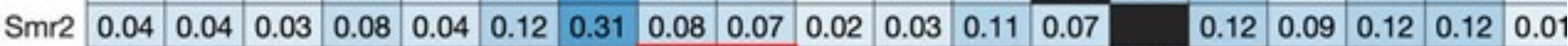

\begin{tabular}{l|l|l|l|l|l|l|l|l|l|l|l|l|l|l|l|l|l|l|l|l} 
Vmp & 0.04 & 0.04 & 0.03 & 0.03 & 0.04 & 0.04 & 0.04 & 0.00 & 0.00 & 0.01 & 0.02 & 0.03 & 0.02 & 0.01 & & 0.06 & 0.07 & 0.09 & $0.0 \varepsilon$ \\
\hline
\end{tabular}

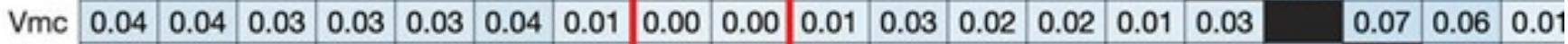

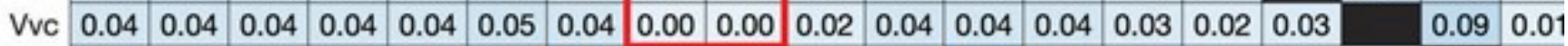

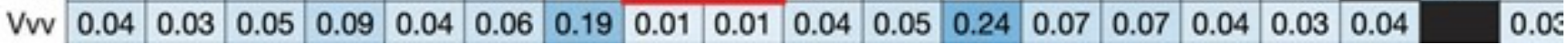

Figure 6. Heat map showing the error rate of the various combination of metrics in classifying the as-built vs. post-processed surfaces. Values of 0 are highlighted.

The upper right portion of Figure 6 shows the results of the LDA classifier, while the lower left shows the results of the NB classifier. When combining two metrics, it is possible to get to a zero error rate using the NB classification, meaning that all of the surfaces were classified correctly. However, Sa and Sz together have a 0.07 error rate for the LDA classification and a 0.04 error rate for the NB classification. Those values are similar to the error rates shown in Figure 5 for single metric classification and is likely due to the strong correlation between the two values. Further improvements are not observed by using both $\mathrm{Sa}$ and $\mathrm{Sz}$ together. However, Sk and Sal both perform well alongside many of the different metrics. Additionally, Vmp (Peak material volume of the scale-limited surface), Vmc (Core material volume of the scale-limited surface), and Vvc (Core void volume of the scale-limited surface) perform well for the NB classification. These metrics are also derived from the bearing area curve and represent the volume of the material in the peaks of the surface, the volume of material in the core region, and volume of the core void region, respectively. Finally, Sdq (Root mean square gradient of the scale-limited surface) and Sdr (Developed interfacial area ratio of the scale-limited surface) also perform well. Sdq represents an average value of the slopes on the surface, while Sdr represents the additional area the surface has compared to a projected area. Additionally, Figure 6 shows that the NB classifier frequently performs better than the LDA classifier. Figure 7 plots some of the metrics for the various surfaces. 

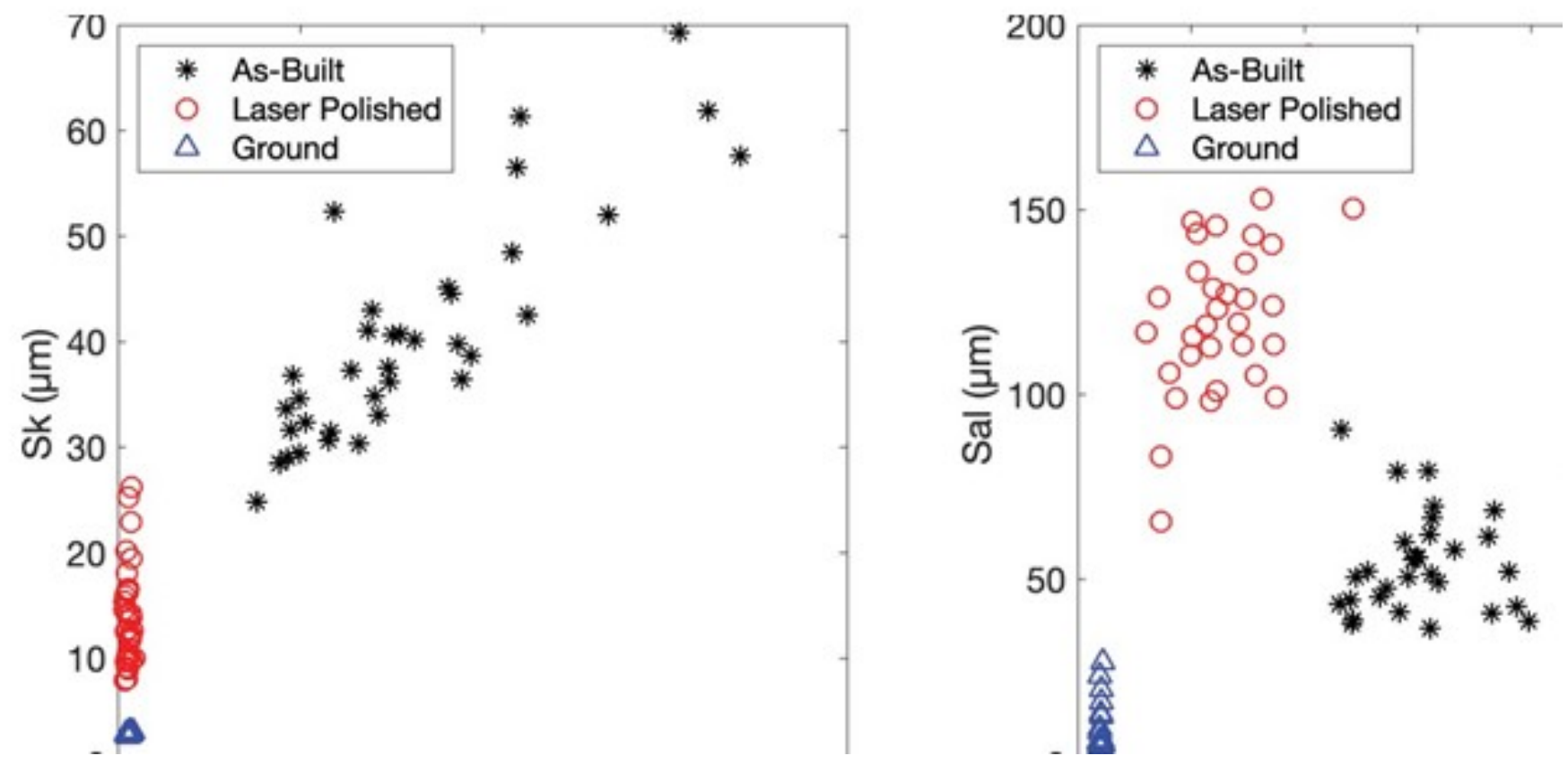

Figure 7. Examples of the distinct grouping of surfaces that occur when (a) Sk and Sdr are used and (b) Sal and Vmc are used.

Figure 7 demonstrates that these metrics distinguish between the various surfaces more clearly than those shown in Figure 4(a) and also shows distinct clustering of the values for the three different surfaces. Additionally, as the variables are not as strongly correlated with one another, more information is shown then when only $\mathrm{Sa}$ and $\mathrm{Sz}$ are reported.

\section{$\underline{\text { As-Built } 90^{\circ} \text { Vertical and } 45^{\circ} \text { Angled Surfaces Classification }}$}

This section uses classification techniques to distinguish between additively manufactured parts built vertically at a 90 and parts built at a 45 angle. As Figure 4(b) shows, these surfaces have similar $\mathrm{Sa}$ and $\mathrm{Sz}$ values to one another. The ability of the individual metrics to distinguish the surfaces is shown in Figure 8. 


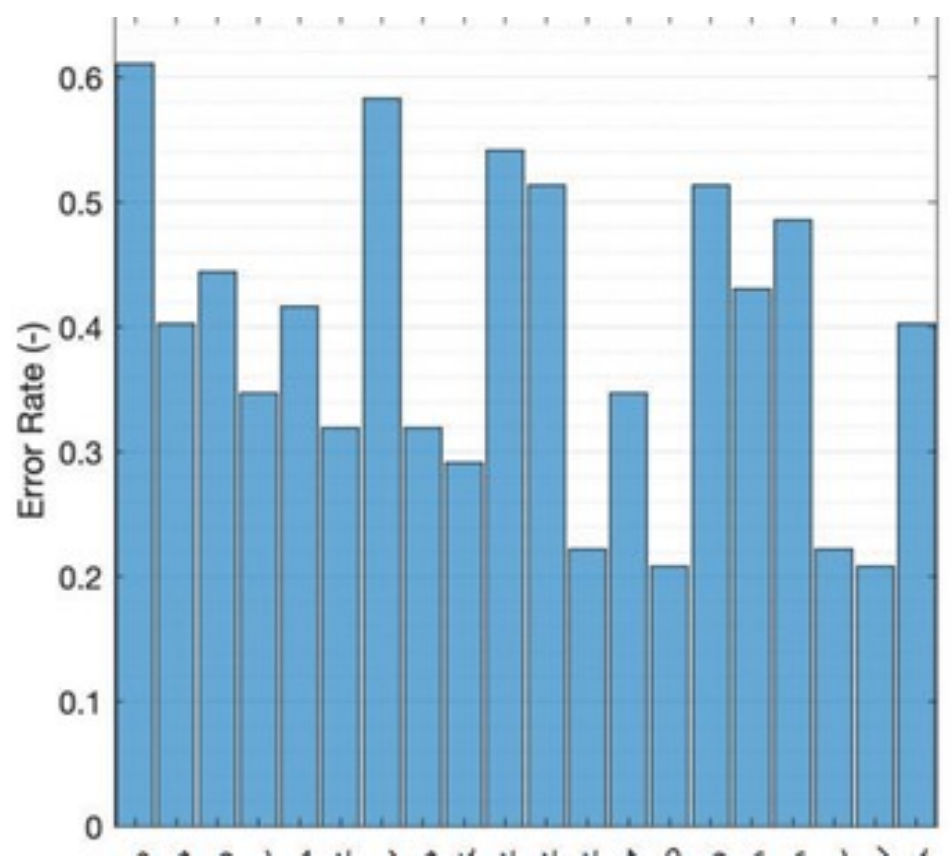

(a)

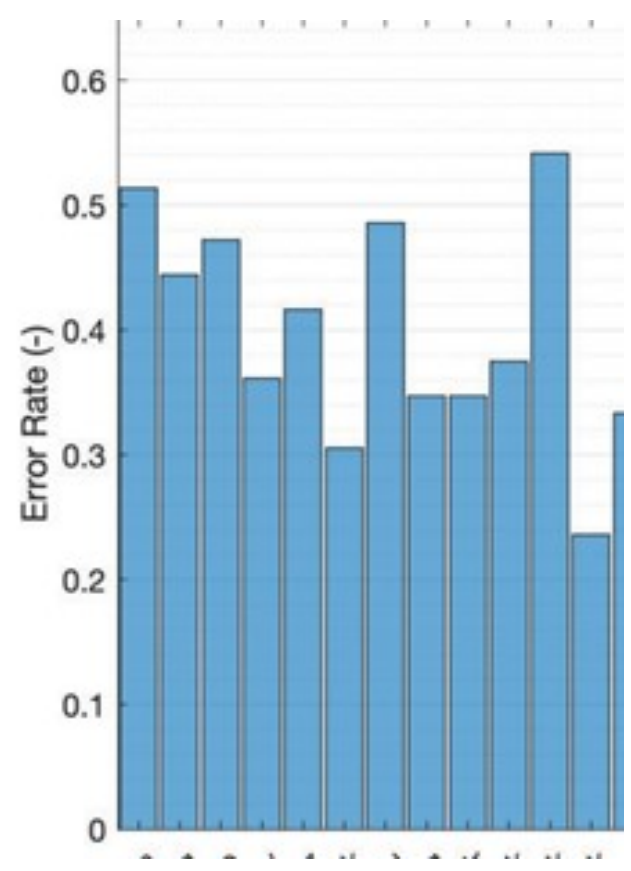

(b)

Figure 8. Error rates for the various metrics at classifying the as-built 90 and as-built 45 vs. surfaces using the (a) LDA classifier and (b) NB classifier.

For these surfaces, Sa and $\mathrm{Sz}$ due a poor job at distinguishing between the groups. Sa has error rates of approximately 0.61 and 0.52 for the LDA classification and NB classification, respectively, while an error rate greater than 0.5 is worse than random chance. Sz has an error rate of approximately 0.42 for both classifications. In contrast, Svk (Reduced dale height), Smr2 (Material ratio 2), Sal, and Vvv (Dale void volume of the scale-limited surface) perform the best, with error rates for all of them between 0.21 and 0.25. Svk, Smr2, and Vvv are derived from the bearing area curve, with Svk representing the height of the valley portion of the surface, Smr2 representing the percentage of the surface that is in the valley portion, and Vvv represents the volume of the valleys from the bearing area curve. The classification ability of the various twometric combinations is shown in Figure 9. 


\begin{tabular}{|c|c|c|c|c|c|c|c|c|c|c|c|c|c|c|c|c|c|c|c|}
\hline & & & & & & & & & & & & & & & & & & & \\
\hline Sa & & 39 & 57 & 0.31 & 0.44 & 0.40 & 0.59 & 0.28 & 0.25 & 0.60 & 0.44 & 0.23 & 0.30 & 0.21 & 0.64 & 0.50 & 0.24 & 0.23 & \\
\hline $\mathrm{Sq}$ & 47 & & & 0.36 & 0.43 & 0.40 & 0.57 & 0.28 & 0.27 & 0.53 & 0.44 & 0.26 & 0.31 & 0.21 & 0.50 & 0.56 & 0.22 & 0.23 & \\
\hline Sp & .52 & 42 & & 0.36 & 0.35 & 0.33 & 0.63 & 0.31 & 0.27 & 0.58 & 0.50 & 0.24 & 0.31 & 0.23 & 0.67 & 0.55 & 0.47 & 0.23 & \\
\hline Sv & 0.37 & 37 & 0.31 & & 0.34 & 0.36 & 0.36 & 0.20 & 0.15 & 0.32 & 0.39 & 0.22 & 0.31 & 0.21 & 0.35 & 0.31 & 0.26 & 0.22 & \\
\hline $\mathrm{Sz}$ & 43 & 44 & 49 & 0.28 & & 0.30 & 0.42 & 0.18 & 0.19 & 0.41 & 0.41 & 0.25 & 26 & 0.17 & 0.40 & 0.42 & 0.33 & 0.21 & \\
\hline Ssk & 32 & 32 & 28 & 0.28 & 0.26 & & 0.22 & 0.33 & 0.32 & 0.35 & 0.40 & 0.25 & 33 & 0.19 & 0.23 & .33 & .38 & .19 & \\
\hline Sku & 0.51 & 51 & 0.50 & 0.36 & 0.49 & 0.25 & & 0.33 & 0.33 & 0.63 & 0.57 & 0.22 & 0.34 & 0.19 & 0.60 & 0.53 & 0.53 & 0.19 & \\
\hline Sdq & 39 & 35 & 0.32 & 0.21 & 0.26 & 0.25 & 0.34 & & 0.30 & 0.23 & 0.35 & 0.18 & 0.31 & 0.23 & 0.33 & 0.27 & 0.31 & 0.15 & \\
\hline Sdr & .38 & 33 & 33 & 0.23 & 0.31 & 0.31 & 0.29 & 0.34 & & 0.18 & 0.33 & 0.19 & 0.31 & 0.22 & 0.31 & 0.20 & 0.31 & 0.14 & \\
\hline Sk & 0.40 & 40 & 40 & 0.38 & 0.39 & 0.33 & 0.39 & 0.36 & 0.38 & & 0.53 & 0.24 & 0.34 & 0.20 & 0.58 & 0.53 & 0.38 & 0.23 & \\
\hline Spk & 1.55 & 0.53 & 0.58 & 0.39 & 0.42 & 0.35 & 0.53 & 0.39 & 0.40 & 0.45 & & 0.26 & 0.33 & 0.20 & 0.40 & 0.47 & 0.44 & 0.23 & \\
\hline Svk & 0.24 & 24 & 0.22 & 0.26 & 0.24 & 0.24 & 0.22 & 0.22 & 0.22 & 0.23 & 0.25 & & 0.23 & 0.22 & 0.24 & 0.22 & .22 & 0.25 & \\
\hline $\mathrm{mr} 1$ & 0.31 & 33 & 0.37 & 0.32 & 0.27 & 0.24 & 0.34 & 0.28 & 0.30 & 0.31 & 0.37 & 0.22 & & 0.24 & 0.33 & 0.32 & 0.35 & 0.22 & \\
\hline Smr2 & 0.24 & 22 & 0.21 & 0.23 & 0.21 & 0.21 & 0.19 & 0.24 & 0.21 & 0.20 & 0.23 & 0.22 & 0.21 & & 0.21 & 0.22 & 0.21 & 0.22 & \\
\hline Vmp & 0.47 & 0.47 & 0.50 & 0.36 & 0.44 & 0.30 & 0.50 & 0.36 & 0.29 & 0.40 & 0.55 & 0.26 & 0.30 & 0.23 & & 0.51 & 0.47 & 0.24 & \\
\hline Vmc & 0.52 & 0.51 & 0.51 & 0. & 0. & 0.31 & 0.53 & 0.36 & 0.33 & 0.42 & 0.55 & 0.24 & 0.33 & 0.23 & 0.49 & & 0.33 & 0.19 & \\
\hline Vvc & 0.50 & 0 & 0.46 & 0. & 0.5 & 0.32 & 0.53 & 0.41 & 0.41 & 0.43 & 0.47 & 0.22 & 0.38 & 0.22 & 0.51 & 0.49 & & 0.19 & \\
\hline Vvv & 0.22 & 0.24 & 0.19 & 0.28 & 0.21 & 0.23 & 0.21 & 0.24 & 0.19 & 0.22 & 0.23 & 0.24 & 0.20 & 0.21 & 0.23 & 0.24 & 0.21 & & \\
\hline
\end{tabular}

Figure 9. Heat map showing the error rate of the various combination of metrics in classifying the two different as-built surfaces. Values of 0.15 and lower are highlighted.

These surfaces are more difficult to distinguish than the as-built vs. post-processed surfaces, and the lowest observable error rate is 0.14 (for the Sdr and Vvv LDA combination). A few metrics stand out as consistently having a lower error rate: Sal, Vvv, Smr2, and Svk. Those metrics are the same metrics that performed well individually. Figure 9 also shows that the LDA classification performs slightly better than the NB method for these surfaces. Two plots showing some examples of the stronger classification combination of metrics are shown in Figure 10.

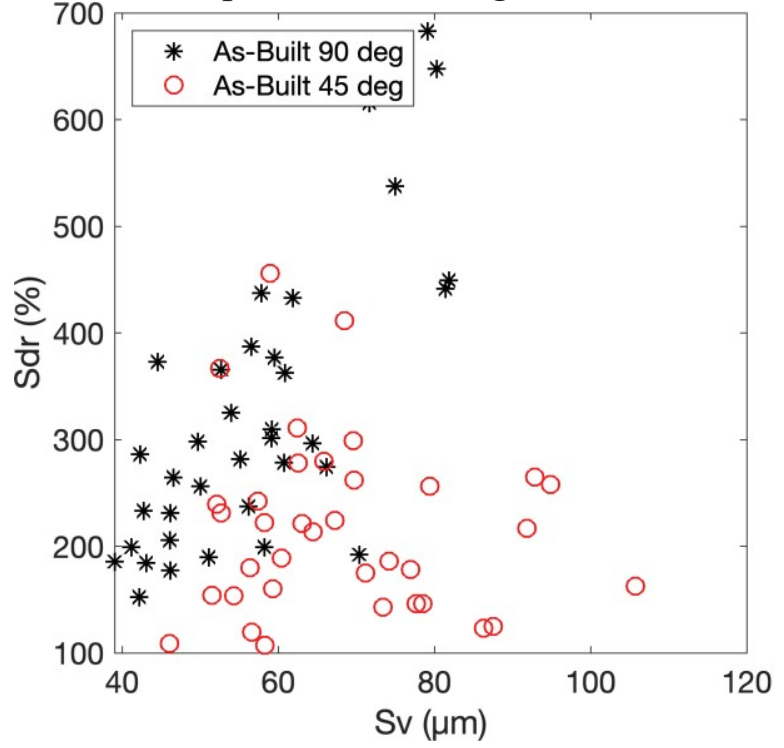

(a)

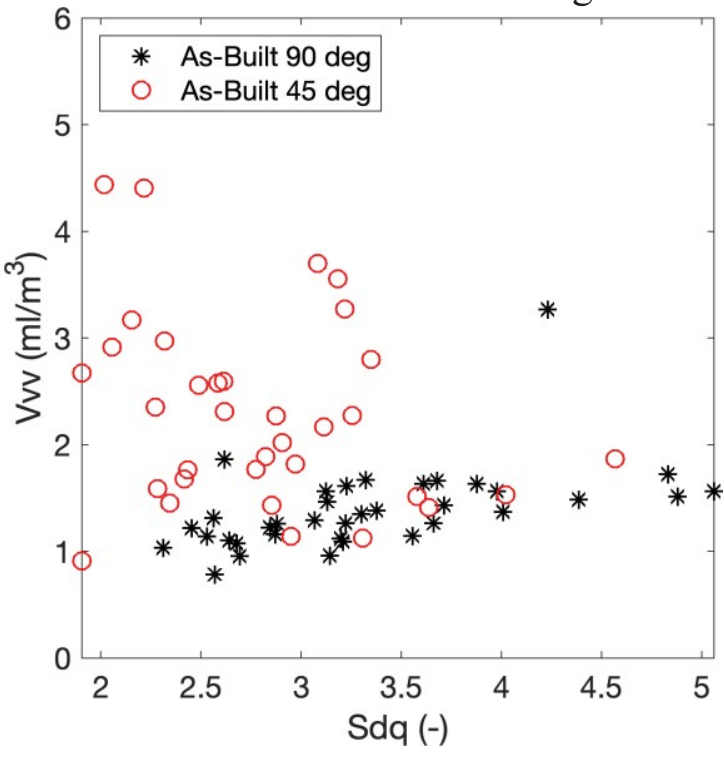

(b)

Figure 10. Examples of the distinct grouping of the as-built surfaces that occur when (a) Sdr and Sv and (b) Sdq and Vvv are used.

In contrast to Figure 4(b), the plots in Figure 10 show more distinct regions where the 90 
and 45 surfaces can be distinguished from one another. These results show that, while using common metrics such as Sa and Sz may imply these surfaces are almost identical, further information and distinctions between the surfaces can be observed when other metrics are used.

\section{Conclusions}

In this work, two different classification techniques are used in two different group classifications of additive manufactured metal parts. The first classification attempted to distinguish between as-built parts, ground parts, and laser polished parts using both a linear discriminatory analysis classifier and a Gaussian naive Bayes classifier. It was found that Sa and Sz performed well as individual metrics for classifying the surfaces with the naive Bayes classifier, but when combinations of metrics were considered, other groupings worked better. Additionally, Sk, Sal, Vmp, Vmc, Vvc, Sdr, and Sdq were all effective at distinguishing between the surfaces when the naive Bayes classifier was used. The second classification tried to differentiate between as-built surfaces that were built at two different angles. For these surfaces, Sa and Sz were not effective at distinguishing between the surfaces, but Svk, Smr2, Sal, and Vvv performed better with error rates of between 0.21 and 0.25 as individual metrics. Additionally, various combinations of metrics were shown to give error rates of 0.15 and low (Sv and Sdr, Sdr and Vvv, Sdr and Vvv, $\mathrm{Vmc}$ and Sal, and Vvc and Sal). These results demonstrate that the metrics with the best ability to distinguish between the various surfaces, and those commonly reported, are not necessarily the same. Especially for additive manufactured parts, whose surfaces are very complex, it is likely worthwhile to report additional metrics when discussing roughness. This work reports various metrics that may be worth considering. It also demonstrates a methodology for determining metrics to use in classifying surfaces, and a similar process could be used to determine whether parts meet specifications, if enough satisfactory parts are available to provide a training data set.

\section{Acknowledgements}

This work was supported by the U.S. National Science Foundation (NSF) grant CMMI1727366 and NSF-supported shared facilities at the University of Wisconsin, as well as by the Deutsche Forschungsgemeinschaft (DFG, German Research Foundation) - 386371584.

\section{$\underline{\text { References }}$}

[1] T. D. Ngo, A. Kashani, G. Imbalzano, K. T. Q. Nguyen, and D. Hui, “Additive manufacturing (3D printing): A review of materials, methods, applications and challenges," Compos. Part B Eng., vol. 143, pp. 172-196, Jun. 2018.

[2] I. Gibson, D.W. Rosen, B. Stucker, Additive Manufacturing Technologies: 3D Printing, Rapid Prototyping, and Direct Digital manufacturing, 2nd., Springer, New York, (2015), pp. 1-498.

[3] B. Rosa, P. Mognol, and J. Hascoët, "Laser polishing of additive laser manufacturing surfaces," J. Laser Appl., vol. 27, no. S2, p. S29102, Feb. 2015.

[4] S. Marimuthu, A. Triantaphyllou, M. Antar, D. Wimpenny, H. Morton, and M. Beard, "Laser polishing of selective laser melted components," Int. J. Mach. Tools Manuf., vol. 95, pp. 97104, Aug. 2015.

[5] W. S. Gora et al., "Enhancing Surface Finish of Additively Manufactured Titanium and Cobalt Chrome Elements Using Laser Based Finishing," Phys. Procedia, vol. 83, pp. 258-263, 2016.

[6] D. Bhaduri et al., "Laser polishing of 3D printed mesoscale components," Appl. Surf. Sci., vol. 405, pp. 29-46, May 2017.

[7] B. Richter, N. Blanke, C. Werner, F. Vollertsen, and F. E. Pfefferkorn, "Effect of Initial 
Surface Features on Laser Polishing of Co-Cr-Mo Alloy Made by Powder-Bed Fusion," JOM, vol. 71, no. 3, pp. 912-919, Mar. 2019.

[8] B. Richter et al., "High-speed X-ray investigation of melt dynamics during continuous-wave laser remelting of selective laser melted Co-Cr alloy," CIRP Ann., May 2019.

[9] J. Delgado, J. Ciurana, and C. A. Rodríguez, "Influence of process parameters on part quality and mechanical properties for DMLS and SLM with iron-based materials," Int. J. Adv. Manuf. Technol., vol. 60, no. 5, pp. 601-610, May 2012.

[10] A. Gebhardt, J.-S. Hötter, and D. Ziebura, "Impact of SLM build parameters on the surface quality," RTejournal - Forum Für Rapid Technol., vol. 2014, no. 1, Jan. 2014.

[11] J. C. Fox, S. P. Moylan, and B. M. Lane, "Effect of Process Parameters on the Surface Roughness of Overhanging Structures in Laser Powder Bed Fusion Additive Manufacturing," Procedia CIRP, vol. 45, pp. 131-134, Jan. 2016.

[12] B. Whip, L. Sheridan, and J. Gockel, "The effect of primary processing parameters on surface roughness in laser powder bed additive manufacturing," Int. J. Adv. Manuf. Technol., May 2019.

[13] A. Townsend, N. Senin, L. Blunt, R. K. Leach, and J. S. Taylor, "Surface texture metrology for metal additive manufacturing: a review," Precis. Eng., vol. 46, pp. 34-47, Oct. 2016.

[14] "ISO 4287:1997 Geometrical Product Specifications (GPS) - Surface texture: Profile method - Terms, definitions and surface texture parameters." International Organization for Standardization, 1997.

[15] "ISO 25178-2:2012 Geometrical Product Specifications (GPS) - Surface texture: Areal - Part 2: Terms, definitions and surface texture parameters." International Organization for Standardization, 2012.

[16] N. Nacereddine and M. Tridi, "Computer-aided shape analysis and classification of weld defects in industrial radiography based invariant attributes and neural networks," in ISPA 2005. Proceedings of the 4th International Symposium on Image and Signal Processing and Analysis, 2005., 2005, pp. 88-93.

[17] R. Vilar, J. Zapata, and R. Ruiz, "An automatic system of classification of weld defects in radiographic images," NDT E Int., vol. 42, no. 5, pp. 467-476, Jul. 2009.

[18] G. Senthil Kumar, U. Natarajan, and S. S. Ananthan, "Vision inspection system for the identification and classification of defects in MIG welding joints," Int. J. Adv. Manuf. Technol., vol. 61, no. 9, pp. 923-933, Aug. 2012.

[19] F. Pernkopf, "Detection of surface defects on raw steel blocks using Bayesian network classifiers," Pattern Anal. Appl., vol. 7, no. 3, pp. 333-342, Sep. 2004.

[20] D.-M. Tsai and C.-F. Tseng, "Surface roughness classification for castings," Pattern Recognit., vol. 32, no. 3, pp. 389-405, Mar. 1999.

[21] P. K. Rao, J. (Peter) Liu, D. Roberson, Z. (James) Kong, and C. Williams, "Online RealTime Quality Monitoring in Additive Manufacturing Processes Using Heterogeneous Sensors,” J. Manuf. Sci. Eng., vol. 137, no. 6, p. 061007, Dec. 2015.

[22] M. Aminzadeh and T. R. Kurfess, "Online quality inspection using Bayesian classification in powder-bed additive manufacturing from high-resolution visual camera images," J. Intell. Manuf., Mar. 2018.

[23] Gibson, I., et al., Materials for Additive Manufacturing, in Additive Manufacturing Technologies. 2021, Springer, Cham. p. 379-428.

[24] J. Ren, S. D. Lee, X. Chen, B. Kao, R. Cheng, and D. Cheung, "Naive Bayes Classification of Uncertain Data," in 2009 Ninth IEEE International Conference on Data Mining, 2009, pp. 944-949. 